\title{
On Validation of Non-Physical Techniques for Elastic Image Registration
}

\author{
Evgeny Gladilin, Karl Rohr and Roland Eils \\ University Heidelberg, IPMB, and German Cancer Research Center, TBI, \\ Im Neuenheimer Feld 580, 69120 Heidelberg \\ Email: e.gladilin@dkfz.de
}

\begin{abstract}
Non-physical techniques for elastic image registration such as different spline-based optimization methods are often applied in biomedical applications for image normalization w.r.t. non-rigid transformations. Since mechanical properties of biological structures to be registered are usually unknown, a "ground truth" validation of the results of image registration is not possible. This article presents a framework for the validation of elastic image registration techniques by a direct comparison of displacement fields vs analytical or numerical reference solutions of customizable boundary value problems. The proposed procedure enables an easy handling of material parameters, domain shapes and boundary conditions, and provides a flexible benchmark-tool for quantitative validation of elastic image registration algorithms.
\end{abstract}

\section{Introduction}

Elastic registration techniques are widely used for normalization of biomedical images with respect to non-rigid transformations (deformations). Depending on core principles utilized for computation of object deformations, these techniques can be formally subdivided into two major groups: physical and non-physical approaches. Low number of required image correspondences, straightforward implementation, computational efficiency and robustness make non-physical registration techniques, such as numerous spline- or optical flow-based methods [1], appear more advantageous for automatic and routine application compared to extensive physical approaches which are based on numerical solving partial differential equations of continuum mechanics. On the other hand, non-physical methods are still expected to produce realistic deformations of biological tissues. Since mechanical properties of biological tissues are highly complex and variable, an exact quantitative validation of the results of image registration by a direct proof of the "ground truth" is usually not possible. In practice, the accuracy of non-rigid registration methods can be benchmarked by exemplary comparison of a non-physical method with (i) physical methods, e.g. finite element methods, or (ii) experimentally assessed deformation fields for some biological or surrogate tissue samples, e.g. the "truth-cube" [2]. However, the results of such validation are firstly limited to that particular BVP which was used for benchmarking or training of an algorithm, and can not be generalized to the cases with different 
material constants, domain shapes, boundary conditions, etc. Further comparative tests with a strongly differing combination of parameters are required to show the ability of a particular method to cope with a more general class of nonrigid registration problems and to prove its accuracy. In this article, a framework for validation of non-physical image registration techniques vs exact analytical or numerical solutions of elastostatic boundary value problems BVPs is presented, which enables a flexible handling of canonical material parameters, boundary conditions and domain shapes for constructing different benchmark-tests.

\section{Methods}

\subsection{Analytical solutions of linear elasticity}

Analytical solution of partial differential equations of elasticity theory is possible only for particularly simple domain shapes and boundary conditions, such as spheric, cylindric, cubic domains or infinite elastic medium. Nevertheless, a boundary value problem for an arbitrarily shaped 3D domain can be constructed using these special closed-form solutions. To demonstrate the idea of this approach, we focus on the fundamental solution of linear elasticity, which describes the response of an infinite 3D elastic medium $\Omega_{\infty}$ with the stiffness $E$ and compressibility $\nu$ to the impact of a point-force $\mathbf{f}(r)=\mathbf{f} \delta(r)[3]$

$$
\mathbf{u}(\mathbf{f}, \mathbf{r})=\frac{1+\nu}{8 \pi E(1-\nu) r}\left((3-4 \nu) \mathbf{f}+\frac{(\mathbf{f} \mathbf{r}) \mathbf{r}}{r^{2}}\right)
$$

where $\mathbf{r}$ is the radial vector from the force application point to an arbitrary observation point. Assume a compact subset $\Omega \subset \Omega_{\infty}$ with the boundary $\Gamma_{\Omega}$, see Fig. 1. In accordance with the Somigliana identity [4], the displacement field $\mathbf{u}_{p}$ for all inner points $p \in \Omega$ of a homogeneous elastic domain (e.g. particular image region) can be computed from the displacements $\mathbf{u}_{q}$ and tractions $\mathbf{t}_{q}$ of the boundary points $q \in \Gamma_{\Omega}$ only [5]

$$
\mathbf{u}_{p}=\int_{\Gamma} \mathbf{G}_{p q} \mathbf{t}_{q} d s-\int_{\Gamma} \mathbf{T}_{p q} \mathbf{u}_{q} d s
$$

whereas $\mathbf{G}_{p q}$ and $\mathbf{T}_{p q}$ are the fundamental solutions for displacements and tractions, respectively. Typically, non-rigid registration is applied for computation of displacements of domain deformations for some predefined boundary correspondences. Thus, we can formulate following procedure for benchmarking a non-rigid registration technique:

- define an arbitrarily shaped spatial domain $\Omega$ with the boundary $\Gamma_{\Omega}$,

- calculate displacements for all points of $\Omega$ and $\Gamma_{\Omega}$ using (1),

- apply your method to compute displacements of inner points $\mathbf{u}_{\Omega}$ for boundary conditions given by the displacements of boundary points $\mathbf{u}_{\Gamma}$,

- compare displacements of inner points obtained with your method vs. reference displacements from (1). 


\subsection{Numerical solutions of elastostatic BVPs}

Following the same basic steps as described above, numerical solutions of arbitrary elastomechanical BVPs obtained for a finite number of mesh nodes can be used for benchmarking a non-rigid image registration algorithm. Using numerical solving techniques such as the finite element method, more complex boundary conditions and material properties can be simulated. We apply the FEM on tetrahedral grids for computation of deformations of of a cubic domain modeled as a St. Venant-Kirchhoff material [6]. Such BVPs resemble the experiments carried out with the "truth cube" and can be seen as its virtual counterpart with homogeneous, isotropic and non-linear elastic material properties.

\subsection{Correlation of vector fields}

For quantification of similarity/dissimilarity between two displacement fields, the normalized scalar product $(N S P)$ can be used

$$
N S P\left(\mathbf{u}_{1}, \mathbf{u}_{2}\right)=\frac{\mathbf{u}_{1} \mathbf{u}_{2}}{\left|\mathbf{u}_{1}\right|\left|\mathbf{u}_{2}\right|}
$$

(3) serves as a descriptor of the relative spatial orientation for each pair of vectors, whereas $N S P=1,0,-1$ stands for correlated, uncorrelated and anticorrelated pairs of vectors, respectively.

\subsection{Topology preservation}

A natural requirement on a realistic material deformation is the preservation of local topology of the registered spatial domain. Violation of the topology preservation by elastic image registration algorithms often occurs when the registration problem is associated with computation of large deformations, and is expressed in penetration of boundaries, distortion of mesh elements, crossing of field lines, etc. Monitoring of the local topology preservation on FE meshes can be done by computing the determinant of the deformation gradient

$$
C=\operatorname{det}(\mathbf{I}+\nabla \mathbf{u})
$$

Topology preservation corresponds to positive $C>0$, while small or negative values $C \leq 0$ indicate extremely deformed or corrupted elements with violated local topology.

\section{Experimental results}

In this section we present some examples of benchmark-tests based on analytical and numerical solutions of elastostatic BVPs, which can be used for the validation of elastic image registration algorithms. 
Fig. 1. Fundamental solution of linear elasticity (1) describes a global displacement field $\mathbf{u}$ in an infinite elastic continuum $\Omega_{\infty}$ induced by a point-force $\mathbf{f}$

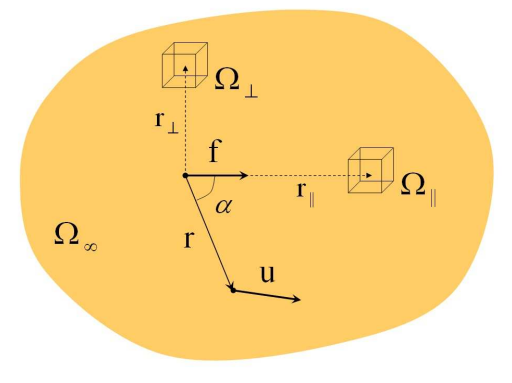

\subsection{Construction of a BVP using the fundamental solution}

Consider a cubic subdomain $\Omega$ of an unloaded infinite elastic medium $\Omega_{\infty}$ with material parameters $E=1$ and $\nu=0.45$. According to (1), a point-force generates a global displacement field in $\Omega_{\infty}$ including boundary and inner points of $\Omega \in \Omega_{\infty}$. Fig. 2 shows the resulting deformation of $\Omega$ for two different cases of its relative orientation w.r.t. the point-force vector, namely $\alpha(\mathbf{r}, \mathbf{f})=\pi$ and $\alpha(\mathbf{r}, \mathbf{f})=\frac{\pi}{2}$. The displacements of boundary points $\mathbf{u}_{\Gamma}$ yield boundary conditions for computation of the displacements of inner points $\mathbf{u}_{\Omega}$ with the subsequent validation of the result vs the reference solution (1). By varying $E$ and $\nu$ in (1), different material properties can be simulated. In order to avoid unnatural effects of the $r^{-1}$ singularity of the Green function, the test-domain $\Omega$ should be placed sufficiently far away from the source point, i.e. $r>>0$. The orientation of $\Omega$ relatively to the force vector $\alpha(\mathbf{r}, \mathbf{f}) \in[0, \pi]$ can be varied to study cross-contraction effects for different values of the Poisson ratio $\nu \in[0,0.5]$.

\subsection{FEM benchmark-tests}

An advantage of numerical solving techniques such as the finite element method is that non-linear material properties and arbitrary complex boundary conditions can be simulated. Smooth displacement fields resulting from closed-form solutions of elasticity theory can, in fact, be approximated by almost any sufficiently smooth spline function. However, non-smooth distributions of deformation energy, which can arise due to geometrical or physical constraints, such as material inhomogeneities in multicomposite biological structures, are more difficult to "mime" with non-physical registration methods. Alternatively to the above described BVPs with the fixed outer boundaries and homogenous onematerial model, we suggest FEM benchmark-tests with mixed boundary conditions, including partially-free and -fixed outer and inner boundaries, as well as non-homogenous material properties. 
Fig. 2. Deformation of a cubic subdomain of an infinite elastic medium $\Omega_{\infty}$ for two different cases of its relative orientation w.r.t. the point-force vector: $\alpha(\mathbf{r}, \mathbf{f})=\pi$ (left) and $\alpha(\mathbf{r}, \mathbf{f})=\frac{\pi}{2}$ (right), respectively
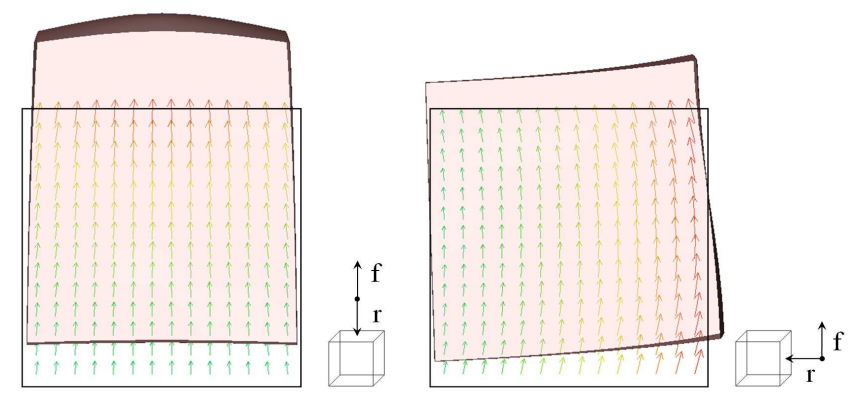

\section{Conclusion}

We have presented a framework for validation of non-rigid image registration techniques using analytical and numerical solutions of customizable elastostatic boundary value problems (BVPs). The proposed validation procedure is based on comparison between theoretically-predicted and simulated displacements of inner domain points computed for predefined boundary displacements. The modeling scheme enables a flexible handling of material properties, domain geometries and boundary conditions for benchmarking non-rigid registration algorithms or fitting free modeling parameters. Numerical criterions for quantification of degree of similarity/dissimilarity between simulated and reference displacements as well as monitoring of local domain topology have been proposed. The presented approach is straightforward in implementation and intends to put the validation of non-rigid registration algorithms vs theoretical solutions of elasticity theory on a more accurate and quantifiable platform.

\section{References}

1. Zitova B, Flusser J. Image registration methods: a survey. Image and Vision Computing 2003;21:977-1000.

2. Kerdok AE, Cotin SM, Ottenmeyer MP, Galea A, Howe RD, Dawson SL. Truth cube: Establishing physical standards for soft tissue simulation. Medical Image Analysis 2003; 7:283-291.

3. Landau LD, Lifschitz EM. Theory of Elasticity. Oxford: Pergamon Press; 1986.

4. Beskos DE. Boundary Element Methods in Mechanics. Amsterdam: North-Holland; 1987.

5. Gladilin E, Pekar V, Rohr K, Stiehl S. A comparison between BEM and FEM for elastic registration of medical images. Image Vision Computing 2006;24(4):375-379.

6. Ciarlet PG. Mathematical Elasticity. Volume I: Three-Dimensional Elasticity. vol. 20 of Studies in Mathematics and its Applications. Amsterdam: North-Holland; 1988. 